\title{
Transport Properties of a Kinetic Model for Chemical Reactions without Barriers
}

\author{
Giselle M. Alves*, Gilberto M. Kremer ${ }^{\dagger}$ and Ana Jacinta Soares** \\ * Escola Técnica, Universidade Federal do Paraná, Curitiba, Brazil \\ ${ }^{\dagger}$ Departamento de Física, Universidade Federal do Paraná, Curitiba, Brazil \\ ** Departamento de Matemática e Aplicações, Universidade do Minho, Braga, Portugal
}

\begin{abstract}
A kinetic model of the Boltzmann equation for chemical reactions without energy barrier is considered here with the aim of evaluating the reaction rate and characterizing the transport coefficient of shear viscosity for the reactive system. The Chapman-Enskog solution of the Boltzmann equation is used to compute the chemical reaction effects, in a flow regime for which the reaction process is close to the final equilibrium state. Some numerical results are provided illustrating that the considered chemical reaction without energy barrier can induce an appreciable influence on the reaction rate and on the transport coefficient of shear viscosity.
\end{abstract}

Keywords: Boltzmann equation, Chemically reactive flows, Transport processes

PACS: $51.10 .+\mathrm{y}, 47.70 . \mathrm{Fw}, 51.10 .+\mathrm{y}$

\section{INTRODUCTION}

Chemical reactions without barriers, that is reactions which proceed without the need of exceeding an activation energy, have become an attractive subject of theoretical and experimental studies, due to their central role in combustion phenomena and in many other processes of astrophysics, organic chemistry, enzymology, chemical physics and biophysics [1]. As discussed in paper [2], the non-barrier approach provides many quantitative chemical information about the reaction mechanism and can be of useful guidance in chemical investigations. In particular, some useful information about the kinetics of the reactions without barriers, improving the research on this subject, can be obtained in the frame of the Boltzmann equation for reactive mixtures. Following this idea, a kinetic model has been proposed in the recent paper [3] to describe a gaseous mixture of two constituents undergoing the chemical reaction $\mathrm{A}+\mathrm{A} \rightleftharpoons \mathrm{B}+\mathrm{B}$, which occur without energy barrier. The fundamental aspect of that non-barrier model is the introduction of a probability coefficient $\chi$ in the reactive collision term and $(1-\chi)$ in the elastic one, which allows to take into account each binary encounter once exactly, either as a reactive or elastic encounter. The coefficient $\chi$ can thus be interpreted as a measure of the reactive degree of the mixture. In particular, the case $\chi=1$ corresponds to consider that all collisions among identical particles occur with chemical reaction whereas $\chi=0$ describes an inert mixture with all collisions being of elastic type.

As well known, there exists a rather vast bibliography within the kinetic theory of chemically reacting gases, starting from the pioneering papers $[4,5]$ by Prigogine and co-workers. In almost papers in existing literature, directed to both theoretical studies and fluid dynamical investigations including reaction effects, the chemical process is described in terms of reactive cross sections with activation energy, so that the large part of the collisions result in elastic scattering, since only few particles can go beyond the activation energy barrier. Furthermore a steric factor connecting the elastic and reactive diameters, normally considered smaller than unity, is also introduced to reduce the number of reactive collisions. On the other hand, some few papers, as for example reference [6,7], consider reactive cross sections without activation energy corresponding to chemical interactions of both Maxwell and hard sphere type. However, the collision terms do not contain any feature which guarantees that each collision contributes exactly once to either elastic or reactive term.

In the present paper, this topic is readdressed, starting from the non-barrier model proposed in paper [3], assuming hard-sphere elastic cross sections and reactive cross sections without activation energy. The Chapman-Enskog method, coupled with Sonine polynomial representation of the one-particle distribution function [8], is applied and an approximate solution of the kinetic equations is explicitly obtained. This non-equilibrium solution contains all the effects induced by the chemical reaction and is used to compute the reaction rate and the transport coefficient of shear viscosity, in a flow regime close to the final stage of the chemical reaction. The non-equilibrium effects induced by the 
reactive process are analyzed in correspondence of the probability coefficient $\chi$ and concentration of the reactants.

\section{PRELIMINARIES OF THE NON-BARRIER KINETIC MODEL}

The non-barrier kinetic model proposed in [3] refers to a binary gaseous mixture whose constituents, denoted by $A$ and $B$, undergo a reversible reaction of the type $\mathrm{A}+\mathrm{A} \rightleftharpoons \mathrm{B}+\mathrm{B}$. Each constituent $\alpha=\mathrm{A}, \mathrm{B}$ has binding energy $\varepsilon_{\alpha}$, molecular mass $m_{\alpha}$ and molecular diameter $d_{\alpha}$, with $m_{A}=m_{B}=m$ and $\mathrm{d}_{A}=\mathrm{d}_{B}=\mathrm{d}$. Examples of such reactions are isomers, in particular the transitions of crotonaldehyde and acrolein (s-cisands-transconformers), the reaction $\mathrm{CH} 3 \mathrm{NC} \rightleftharpoons \mathrm{CH} 3 \mathrm{CN}$ and the transformation cyclopropane $\rightleftharpoons$ propene.

The affinity $\mathcal{A}$ of the chemical reaction is defined in terms of the chemical potential $\mu_{\alpha}$ of the constituents by

$$
\mathcal{A}=2\left(\mu_{A}-\mu_{B}\right), \quad \text { with } \quad \mu_{\alpha}=\varepsilon_{\alpha}-k T\left[\frac{3}{2} \ln T-\ln n_{\alpha}+\mathcal{C}\right],
$$

where $k$ is the Boltzmann constant, $T$ the temperature of the whole mixture, $n_{\alpha}$ the particle number density of the constituent $\alpha$, and $\mathcal{C}$ denotes a real constant. From the definitions (1) one obtains

$$
\mathcal{A}=-\mathcal{Q}+2 k T \ln \left(\frac{n_{A}}{n_{B}}\right), \quad \text { where } \quad \mathcal{Q}=2\left(\varepsilon_{B}-\varepsilon_{A}\right) .
$$

Above $\mathcal{Q}$ denotes the reaction heat which is defined as the difference between the binding energies of the products and reactants. The reaction is exothermic when $\mathcal{Q}<0$ and endothermic when $\mathcal{Q}>0$. In chemical equilibrium the concentrations are constrained by the mass action law, namely,

$$
e^{-\mathcal{Q}^{\star}}=\left(\frac{n_{B}^{\mathrm{eq}}}{n_{A}^{\mathrm{eq}}}\right)^{2}, \quad \text { with } \quad \mathcal{Q}^{\star}=\frac{\mathcal{Q}}{k T_{\mathrm{eq}}},
$$

where $T_{\mathrm{eq}}$ is the equilibrium temperature of the mixture. The gas molecules undergo binary elastic collisions and binary reactive interactions and, as usual, the elastic collisions assure momentum and kinetic energy conservation whereas the reactive interactions preserve momentum and total energy, which includes the binding energy of the molecules. In the phase space the state of the reactive mixture is characterized by the distribution function $f_{\alpha}\left(\mathbf{x}, \mathbf{c}_{\alpha}, t\right)$, whose space-time evolution is described by the Boltzmann equation (see paper [3])

$$
\begin{aligned}
\frac{\partial f_{\alpha}}{\partial t}+c_{i}^{\alpha} \frac{\partial f_{\alpha}}{\partial x_{i}}=\sum_{\beta=A}^{B}(1- & \left.\chi \delta_{\alpha \beta}\right) \int\left[f_{\alpha}^{\prime} f_{\beta}^{\prime}-f_{\alpha} f_{\beta}\right] g_{\beta \alpha} \sigma_{\alpha \beta} d \Omega d \mathbf{c}_{\beta} \\
& +\chi \int\left[f_{\gamma} f_{\gamma_{1}}-f_{\alpha} f_{\alpha_{1}}\right] \sigma_{\alpha}^{\star} g_{\alpha} d \Omega^{\star} d \mathbf{c}_{\alpha_{1}}, \quad \alpha \neq \gamma=A, B .
\end{aligned}
$$

The first term on the right hand side of the above equation refers to elastic collisions and the second one corresponds to chemical interactions. The pre, post and relative elastic collisional velocities are denoted by $\left(\mathbf{c}_{\alpha}, \mathbf{c}_{\beta}\right),\left(\mathbf{c}_{\alpha}^{\prime}, \mathbf{c}_{\beta}^{\prime}\right)$ and $\mathbf{g}_{\beta \alpha}=\mathbf{c}_{\beta}-\mathbf{c}_{\alpha}$, respectively, $\delta_{\alpha \beta}$ is the Kronecker symbol, $\sigma_{\alpha \beta}$ is the elastic differential cross section and $d \Omega$ refers to an element of solid angle which characterizes the elastic scattering process. Furthermore, $\left(\mathbf{c}_{A}, \mathbf{c}_{A_{1}}\right)$ and $\left(\mathbf{c}_{B}, \mathbf{c}_{B_{1}}\right)$ are the velocities of the reactants and products of the forward reaction, respectively, and the sub-index 1 is used to distinguish two identical molecules that participate in the reactive collision, $\sigma_{\alpha}^{\star}$ denotes the reactive differential cross section, $d \Omega^{\star}$ the element of solid angle and $\mathbf{g}_{\alpha}=\mathbf{c}_{\alpha_{1}}-\mathbf{c}_{\alpha}$ the relative velocity. Moreover, the parameter $\chi$, with $0 \leq \chi \leq 1$, is a scalar factor which represents the probability of a chemical binary encounter among identical molecules. In the case $\alpha=\beta$, the term $(1-\chi)$ is a reduced factor for the elastic contributions among identical molecules which avoids a double counting in the sense that each encounter among identical molecules gives only one contribution to equation (4), which can be either of elastic or reactive type. As previously mentioned in the introduction, this is not the usual procedure in the literature of the Boltzmann equation for chemically reacting mixtures, since the majority of the research papers deal with reactive cross sections with activation energy and introduce a steric factor connecting elastic and reactive interaction diameters. Therefore, reactive collisions occur only for those pairs of molecules with relative translational energy larger than the activation energy, whereas almost elastic collisions occur for low energy levels. In this sense there exists some overlapping for high energies, since each encounter between particles of the same constituent is counted both as an elastic collision and as a reactive interaction. 
At the molecular level, the collisional dynamics of the considered gas is described in terms of cross sections of hard-sphere type, i.e., $\sigma_{\alpha \beta}=\mathrm{d}^{2} / 4$ for elastic scattering and $\sigma_{\alpha}^{*}=\mathrm{d}^{2} / 4$ for reactive interactions. On the other hand, the macroscopic picture is described by the balance equations for the basic fields of the constituent particle number densities $n_{\alpha}$, mean velocity $v$ and temperature $T$ of the whole mixture, defined in terms of the distribution functions by

$$
n_{\alpha}=\int f_{\alpha} d \mathbf{c}_{\alpha}, \quad v_{i}=\frac{1}{\rho} \sum_{\alpha=A}^{B} \int m c_{i}^{\alpha} f_{\alpha} d \mathbf{c}_{\alpha}, \quad T=\frac{1}{3 n k} \sum_{\alpha=A}^{B} \int m \xi_{\alpha}^{2} f_{\alpha} d \mathbf{c}_{\alpha},
$$

where

$$
\rho=\sum_{\alpha=A}^{B} \rho_{\alpha}, \quad \rho_{\alpha}=m n_{\alpha}, \quad n=\sum_{\alpha=A}^{B} n_{\alpha}, \quad \xi_{i}^{\alpha}=c_{i}^{\alpha}-v_{i} .
$$

The balance equations for the fields (5) are

$$
\begin{aligned}
& \frac{\partial n_{\alpha}}{\partial t}+\frac{\partial}{\partial x_{i}}\left(n_{\alpha} u_{i}^{\alpha}+n_{\alpha} v_{i}\right)=\mathcal{J}^{\alpha}, \quad \mathcal{J}^{\alpha}=\chi \int\left[f_{\gamma} f_{\gamma_{1}}-f_{\alpha} f_{\alpha_{1}}\right] \sigma_{\alpha}^{\star} g_{\alpha} d \Omega^{\star} d \mathbf{c}_{\alpha_{1}} d \mathbf{c}_{\alpha}, \\
& \frac{\partial \rho v_{i}}{\partial t}+\frac{\partial}{\partial x_{j}}\left(p_{i j}+\rho v_{i} v_{j}\right)=0, \\
& \frac{\partial}{\partial t}\left[\frac{3}{2} n k T+\sum_{\alpha=A}^{B} n_{\alpha} \varepsilon_{\alpha}+\frac{1}{2} \rho v^{2}\right]+\frac{\partial}{\partial x_{i}}\left[q_{i}+p_{i j} v_{j}+\left(\frac{3}{2} n k T+\sum_{\alpha=A}^{B} n_{\alpha} \varepsilon_{\alpha}+\frac{1}{2} \rho v^{2}\right) v_{i}\right]=0,
\end{aligned}
$$

where $u_{i}^{\alpha}$ is the constituent diffusion velocity, $p_{i j}$ and $q_{i}$ are pressure tensor and heat flux of the whole mixture:

$$
u_{i}^{\alpha}=\int \xi_{i}^{\alpha} f_{\alpha} d \mathbf{c}_{\alpha}, \quad p_{i j}=\sum_{\alpha=A}^{B} \int m \xi_{i}^{\alpha} \xi_{j}^{\alpha} f_{\alpha} d \mathbf{c}_{\alpha}, \quad q_{i}=\sum_{\alpha=A}^{B} \int \frac{m}{2} \xi_{\alpha}^{2} \xi_{i}^{\alpha} f_{\alpha} d \mathbf{c}_{\alpha} .
$$

\section{NONEQUILIBRIUM DISTRIBUTION FUNCTION}

The nonequilibrium distribution function is here obtained by using the Chapman-Enskog method discussed in many papers about chemically reacting gases (see, for example, paper [8]). The chemical regime of the considered gas is the one of the final stage of the reaction process, when the chemical affinity $\mathcal{A}$ is a small quantity and the relaxation times of both chemical reaction and elastic scattering are of the same order. The nonequilibrium distribution function is searched as a small deviation $\Phi_{\alpha}^{(1)}$ of the Maxwellian distribution $f_{\alpha}^{M}$ of mechanical equilibrium only, that is

$$
f_{\alpha}^{(1)}=f_{\alpha}^{M}\left[1+\Phi_{\alpha}^{(1)}\right], \quad f_{\alpha}^{M}=n_{\alpha}^{\mathrm{eq}}\left(\frac{m}{2 \pi k T}\right)^{\frac{3}{2}} \exp \left(-\frac{m \xi_{\alpha}^{2}}{2 k T}\right),
$$

$n_{\alpha}^{\mathrm{eq}}$ being the equilibrium number densities which are constrained by the mass action law (3). The deviations $\Phi_{\alpha}^{(1)}$ from the equilibrium are explicitly computed, after inserting the expansions (11) into the Boltzmann equation (4), neglecting the non-linear terms in the deviations and keeping only the derivatives of the Maxwellian distribution on the left hand side of the equation, since these derivatives are the thermodynamic forces that induce the appearance of the considered deviations. Proceeding with the usual steps of the Chapman Enskog method, the deviations are represented in terms of the Sonine polynomials $\mathcal{S}_{m}^{(r)}$ in the form

$$
\Phi_{\alpha}^{(1)}=-\sum_{n=0}^{1} \mathrm{a}_{n}^{\alpha} \mathcal{S}_{3 / 2}^{(n)}\left(\mathcal{C}_{\alpha}^{2}\right) \xi_{i}^{\alpha} \frac{1}{T} \frac{\partial T}{\partial x_{i}}-\mathrm{b}_{0}^{\alpha} \mathcal{S}_{5 / 2}^{(0)}\left(\mathcal{C}_{\alpha}^{2}\right) \xi_{\langle i}^{\alpha} \xi_{j\rangle}^{\alpha} \frac{m}{k T} \frac{\partial v_{\langle i}}{\partial x_{j\rangle}}-\sum_{n=0}^{1} \mathrm{~d}_{n}^{\alpha} \mathcal{S}_{3 / 2}^{(n)}\left(\mathcal{C}_{\alpha}^{2}\right) \xi_{i}^{\alpha} d_{i}^{A}-\mathrm{e}_{1}^{\alpha} \mathcal{S}_{1 / 2}^{(1)}\left(\mathcal{C}_{\alpha}^{2}\right) \frac{\mathcal{A}}{k T}
$$

Above, $\mathcal{C}_{\alpha}^{2}=m \xi_{\alpha}^{2} / 2 k T$ are dimensionless quantities, $\mathrm{a}_{n}^{\alpha}, \mathrm{b}_{0}^{\alpha}, \mathrm{d}_{n}^{\alpha}$ and $\mathrm{e}_{1}^{\alpha}$ are coefficients which depend on $n_{\alpha}$ and $T$ and are determined from the Boltzmann equation. The traceless part of the gradient of velocity, $\partial v_{\langle i} / \partial x_{j\rangle}$, the temperature gradient $\partial T / \partial x_{i}$, the affinity $\mathcal{A} / k T$ and the generalized diffusion force $d_{i}^{A}$ represent the thermodynamic forces. The generalized diffusion force is given by $d_{i}^{A}=\partial x_{A} / \partial x_{i}=-\partial x_{B} / \partial x_{i}=-d_{i}^{B}$, with $x_{\alpha}=n_{\alpha} / n$ being the molar fraction of the constituent $\alpha$. 
After some standard but rather cumbersome calculations, the deviations $\Phi_{\alpha}^{(1)}$ have been explicitly computed so that the nonequilibrium distribution functions $f_{\alpha}^{(1)}$ are completely specified. The detailed expression for $f_{\alpha}^{(1)}$ is here omitted for brevity. It depends on the probability coefficient $\chi$ and contains all the information about the deviations of the reactive system from the equilibrium.

\section{REACTION RATE AND SHEAR VISCOSITY}

The nonequilibrium effects due to the presence of chemical process and the influence of the chemical reaction on the transport process can be evaluated starting from the distribution function determined as described in the previous section. In the present paper, the analysis will be restricted to the the transport coefficient of shear viscosity and to the influence of the probability coefficient $\chi$ on the reaction rate.

Navier-Stokes Law. The constitutive equation for the pressure tensor $p_{i j}$ is obtained by inserting first the nonequilibrium distribution (11), with the deviation $\Phi_{\alpha}^{(1)}$ characterized as described in the previous section, into its definition $(10)_{2}$. The integration of the resulting equation leads to the Navier-Stokes law of the mixture, namely

$$
p_{i j}=p \delta_{i j}-2 \mu \frac{\partial v_{\langle i}}{\partial x_{j\rangle}}
$$

where $p$ and $\mu$ are the pressure and shear viscosity coefficient, respectively, of the mixture, namely

$$
p=\frac{1}{3} \sum_{\alpha=A}^{B} \int m \xi_{\alpha}^{2} f_{\alpha}^{(0)} d \mathbf{c}=\sum_{\alpha=A}^{B} n_{\alpha} k T \quad \text { and } \quad \mu=\sum_{\alpha=A}^{B} \int m \xi_{i}^{\alpha} \xi_{j}^{\alpha} f_{\alpha}^{(0)} \Phi_{\alpha}^{(1)} d \mathbf{c}=\sum_{\alpha=A}^{B} n_{\alpha} \mathrm{b}_{0}^{\alpha} k T .
$$

The above expressions have the same qualitative form as the corresponding ones in paper [8], for example, but the coefficient $b_{0}^{\alpha}$, which figures in the expression $(14)_{2}$, depends now on the probability coefficient $\chi$. This explicit dependence is crucial for the investigation of the influence of the chemical reaction on the transport process and this will lead to a new contribution in the research of the chemically reactive gases.

Reaction Rate. When the system is an inert gas, the number of the molecules of each constituent always remains constant, even if the system is in some non-equilibrium state. Conversely, in the case of reactive systems, the chemical reaction induces a variation of the number of the molecules of each constituent and the corresponding production rate density is given by the term $\mathcal{J}^{\alpha}$ on the right hand side of the balance equation $(7)_{1}$. For a binary system, if the $A$-constituent has a positive production, this implies that the $B$-constituent is being consumed by the reaction. So it is necessary only to know the behavior of only one constituent, for example $A$. The variation of the $A$-constituent results from the net balance between what is produced by the reverse reaction $A+A \leftarrow B+B$ and what is consumed by the direct reaction $A+A \rightarrow B+B$, given by $\mathcal{J}_{\mathrm{r}}^{A}$ and $\mathcal{J}_{\mathrm{d}}^{A}$, namely

$$
\mathcal{J}^{A}=\mathcal{J}_{\mathrm{r}}^{A}-\mathcal{J}_{\mathrm{d}}^{A}, \quad \mathcal{J}_{\mathrm{r}}^{A}=\chi \int f_{B} f_{B_{1}} g_{B} \sigma_{B}^{\star} d \Omega^{\star} d \mathbf{c}_{B_{1}} d \mathbf{c}_{B}, \quad \mathcal{J}_{\mathrm{d}}^{A}=\chi \int f_{A} f_{A_{1}} g_{A} \sigma_{A}^{\star} d \Omega^{\star} d \mathbf{c}_{A_{1}} d \mathbf{c}_{A}
$$

By substituting the distribution function (11) into the expressions (15) and then performing the integration, we get

$$
\mathcal{J}_{\mathrm{r}}^{A}=n_{A}^{2} \kappa^{(0)}\left[1-\left(1-\kappa_{r}^{\star}\right) \frac{\mathcal{A}}{k T}\right] \quad \text { and } \quad \mathcal{J}_{\mathrm{d}}^{A}=n_{A}^{2} \kappa^{(0)}\left[1+\kappa_{d}^{\star} \frac{\mathcal{A}}{k T}\right],
$$

where $\kappa^{(0)}$ is the first approximation to the rate constant, $\boldsymbol{\kappa}_{d}^{\star}$ and $\boldsymbol{\kappa}_{r}^{\star}$ are the dimensionless second approximations to the direct and reverse rate constants, respectively, given by

$$
\kappa^{(0)}=4 \chi \mathrm{d}_{\mathrm{R}}^{2} \sqrt{\frac{\pi k T}{m}}, \quad \kappa_{r}^{\star}=\frac{\kappa_{r}^{(1)}}{\kappa^{(0)}}=\left(\frac{1}{2}-\mathcal{Q}^{\star}\right) \mathrm{e}_{1}^{B}, \quad \kappa_{d}^{\star}=\frac{\kappa_{d}^{(1)}}{\kappa^{(0)}}=\frac{1}{2} \mathrm{e}_{1}^{A} .
$$

It is interesting to note that the coefficient $\kappa^{(0)}$, apart from the probability coefficient $\chi$, is the same as the preexponential factor of the Arrhenius equation. However, the important point here is that the coefficient $\kappa^{(0)}$ is in fact the actual rate of reaction in the first order approximation, since in the case of non-barrier model there is no activation energy and the rate constants of the direct and reverse reactions have - in this approximation - the same probability. It is important to underline that the coefficients $e_{1}^{A}$ and $e_{1}^{B}$ depend explicitly on the probability coefficient $\chi$ and are related by the constraint $n_{A} \mathrm{e}_{1}^{A}=-n_{B} \mathrm{e}_{1}^{B}$. 


\section{RESULTS AND DISCUSSION}

In order to illustrate the behavior of the shear viscosity coefficient $\mu$ and of the rate constant $\kappa_{d}^{\star}$ defined in the previous section, let us introduce first the dimensionless coefficient $\mu^{\star}$ given by

$$
\mu^{\star}=\frac{\mu}{\mu_{I}}, \quad \text { with } \quad \mu_{I}=\frac{5}{16} \frac{1}{\mathrm{~d}^{2}} \sqrt{\frac{m k T}{\pi}},
$$

where $\mu_{I}$ represents the coefficient of shear viscosity of a single inert gas. After the introduction of dimensionless coefficients $\kappa_{d}^{\star}, \kappa_{r}^{\star}$ and $\mu^{\star}$, there remain three parameters to be considered before analyzing the behavior of the variables: the molar fraction $x_{A}$, the probability coefficient $\chi$ and the dimensionless reaction heat $\mathcal{Q}^{\star}=\mathcal{Q} / k T$. With respect to the last one, it is directly connected with the equilibrium molar fractions, through the mass action law (3) 1

$$
\mathrm{e}^{\mathcal{Q}^{\star}}=\frac{x_{A}^{2}}{\left(1-x_{A}\right)^{2}}
$$

so that the number of parameters reduces to two independent ones, namely $x_{A}$ and $\chi$. In the present work, both types of encounters (elastic and reactive) are considered to have the same order of magnitude. So we choose values for the parameter $\chi$ between 0 (absence of reactive collisions or chemical reactions) and 0.5 (each encounter among identical particles results in both a reactive interaction or an elastic encounter with the same probability). Concerning the molar fraction $x_{A}$, we will restrict $x_{A}$ to the range $0.3 \leq x_{A} \leq 0.7$, which represent mixtures where the proportion of the two constituents are not too disparate.

Figures 1 and 2 describe the behavior of the dimensionless shear viscosity coefficient $\mu^{*}$ and direct reaction rate $\kappa_{d}^{\star}$ as functions of the molar fraction $x_{A}$ and probability coefficient $\chi$. From Figure 1 we can observe that there is an inflection point common to all curves for different values of $\chi$, when the molar fractions of both constituents are equal, i.e., $x_{A}=x_{B}=0.5$. This point corresponds to a mixture with vanishing reaction heat, see Eq. (19), and separates the region $x_{A}<0.5$ on its left hand side, with negative reaction heat, from the region $x_{A}>0.5$ on the right hand side, with positive reaction heat. Moreover, for the considered non-barrier model, the constituents are distinguished by the formation energies only, which are related to the reaction heat by $\mathcal{Q}=2\left(\varepsilon_{B}-\varepsilon_{A}\right)$. Therefore, the inflection point corresponds to a mixture for which the particles $A$ and $B$ become indistinguishable and the reaction process does not affect the chemical composition of the gas system. On the other hand, the region $x_{A}<0.5$ corresponds to a mixture with predominant $B$-constituent and exothermic chemical reaction whereas the region $x_{A}>0.5$ to a mixture with predominant $A$-constituent and endothermic chemical reaction.

Other main observations that we can make here are the following:

i) It is noticeable that the effect on the shear viscosity ratio $\mu^{*}$ is larger when the probability of reactive collision tends to 0.5 (more reactions occur, larger is the effect);

ii) When there are no reactive collisions $(\chi=0)$, the shear viscosity ratio $\mu^{\star}$ remains constant and equal to one for any value of the molar fraction $x_{A}$, meaning that the reactive shear viscosity coefficient $\mu$ is equal to the inert one, as expected, since, in this case, there are no reactive collisions, just elastic ones.

iii) For $x_{A}=x_{B}=0.5$ the reaction heat vanishes and there is no any reactive effect on the shear viscosity ratio $\mu^{*}$;

iv) When $x_{A}<0.5$, the system becomes more viscous due to the chemical reaction; this feature can be explained as follows: since the reaction heat is negative, the energy is released (exothermic process), so that the temperature of the mixture increases and causes a rise in the shear viscosity of the mixture. Conversely, when we analyze the right side of inflection point on Figure 1, just the opposite occurs: there are more $A$-particles than $B$-particles and the shear viscosity decreases as a consequence of the temperature decreasing in an endothermic process.

With respect to the dimensionless reaction rate $\kappa^{\star}$, its first approximation $\kappa^{(0)}$ depends only on the probability coefficient $\chi$. This result is different from the one when an activation energy is involved and the Arrhenius equation is valid. Moreover, the second approximation of $\kappa^{\star}$ is a function of both the molar fraction $x_{A}$ and the probability coefficient $\chi$, through the coefficients $\mathrm{e}_{1}^{A}$ and $\mathrm{e}_{1}^{B}$. The behavior of the second approximation for the direct reaction rate $\kappa_{d}^{\star}$ is plotted in Figure 2 as function of the molar fraction $x_{A}$. Here we also note that this coefficient tends to zero when the probability coefficient is zero or when there is equal amount of $A$ and $B$-particles. Again, the largest effect is observed when the probability coefficient $\chi$ assumes its larger value, here $\chi=0.5$. This behavior is totally expected, since under this condition the reactions become more frequent. 


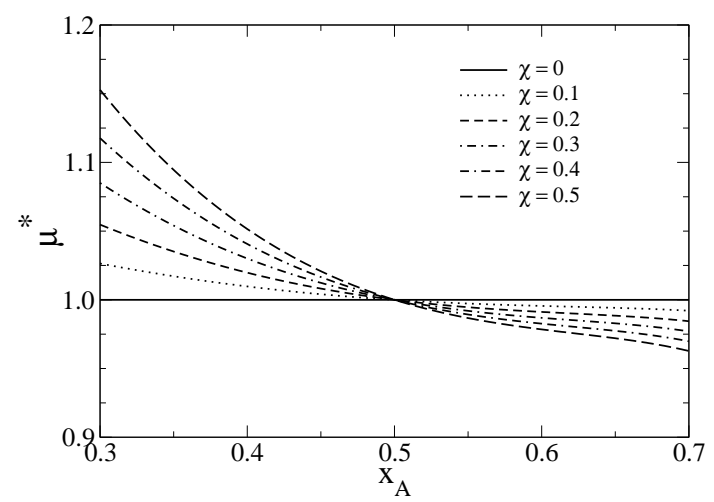

FIGURE 1. Evolution of dimensionless shear viscosity coefficient $\mu^{*}$ as function of the molar fraction $x_{A}$, for different values of the probability coefficient $\chi$.

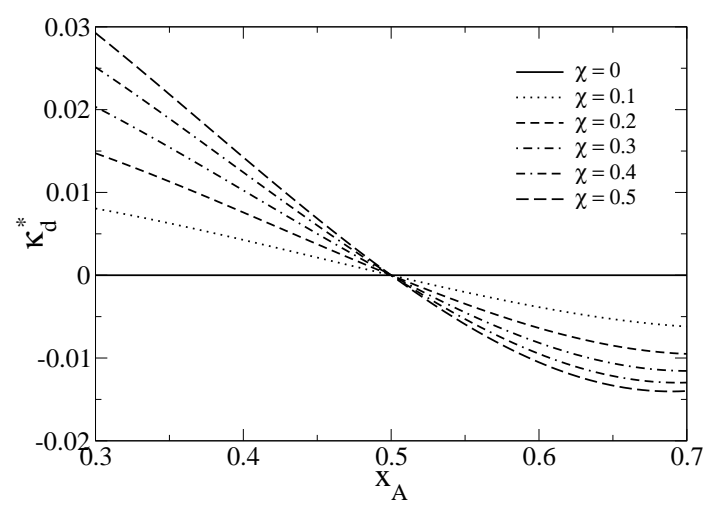

FIGURE 2. Evolution of direct reaction rate $\kappa_{d}^{\star}$ as function of the molar fraction $x_{A}$, for different values of the probability coefficient $\chi$.

\section{ACKNOWLEDGMENTS}

The paper is partially supported by Brazilian Research Council (CNPq), by Minho University Mathematics Centre (CMAT-FCT) and by Project FCT-PTDC/MAT/68615/2006.

\section{REFERENCES}

1. J. P. Hessler, J. Chem Phys., 111, 4068-4076 (1999).

2. J. P. Guthrie and V. Pitchko, J. Phys. Org. Chem., 17, 548-559 (2004).

3. G. M. Kremer and A. J. Soares, "A kinetic model for chemical reactions without barriers", in Rarefied Gas Dynamics, edited by Akashi Abe, AIP Conference Proceedings 1084, American Institute of Physics, New York, 2009, pp. 105-110.

4. I. Prigogine and E. Xhrouet, Physica , 15, 913-933 (1949).

5. I. Prigogine and M. Mahieu, Physica, 16, 51-64 (1950).

6. M. Bisi, M. Groppi and G. Spiga, Continuum Mech. Thermodyn., 14, 207-222 (2002).

7. B. D. Shizgal and A. Chikhaoui, Physica A 365, 317-332 (2006).

8. G. M. Alves and G. M. Kremer, J. Chem Phys., 117, 2205-2215 (2002). 